\title{
The contribution of a 9p21.3 variant, a KIF6 variant, and C-reactive protein to predicting risk of myocardial infarction in a prospective study
}

\author{
Dov Shiffman ${ }^{1 *}$, Ellen S O'Meara ${ }^{2}$, Charles M Rowland ${ }^{1}$, Judy Z Louie ${ }^{1}$, Mary Cushman ${ }^{3}$, Russell P Tracy ${ }^{4}$, \\ James J Devlin', Bruce M Psaty ${ }^{5}$
}

\begin{abstract}
Background: Genetic risk factors might improve prediction of coronary events. Several variants at chromosome 9p21.3 have been widely reported to be associated with coronary heart disease (CHD) in prospective and casecontrol studies. A variant of KIF6 (719Arg) has also been reported to be associated with increased risk of CHD in large prospective studies, but not in case-control studies. We asked whether the addition of genetic information (the 9p21.3 or KIF6 variants) or a well-established non-genetic risk factor (C-reactive protein [CRP]) can improve risk prediction by the Framingham Risk Score (FRS) in the Cardiovascular Health Study (CHS)-a prospective observational study of risk factors for cardiovascular disease among > 5,000 participants aged 65 or older.
\end{abstract}

Methods: Improvement of risk prediction was assessed by change in the area under the receiver-operator characteristic curve (AUC) and by net reclassification improvement (NRI).

Results: Among white participants the FRS was improved by addition of KIF6 719Arg carrier status among men as assessed by the AUC (from 0.581 to $0.596, P=0.03$ ) but not by NRI (NRI $=0.027, P=0.32$ ). Adding both CRP and $719 \mathrm{Arg}$ carrier status to the FRS improved risk prediction by the AUC $(0.608, P=0.02)$ and $\mathrm{NRI}(0.093, \mathrm{P}=0.008)$ in men, but not women ( $P \geq 0.24)$.

Conclusions: While none of these risk markers individually or in combination improved risk prediction among women, a combination of KIF6 719Arg carrier status and CRP levels modestly improved risk prediction among white men; although this improvement is not significant after multiple-testing correction. These observations should be investigated in other prospective studies.

\section{Background}

The Framingham Risk Score (FRS) is a risk prediction model developed by the Framingham investigators to predict the probability of developing coronary heart disease (CHD) [1]. This risk prediction model calculates the probability of a CHD event over a given time period for men and women separately by integrating information about traditional risk factors for CHD, including age, blood pressure, low-density lipoprotein cholesterol (LDL-C), high-density lipoprotein cholesterol (HDL-C), smoking behavior, and diabetes status. The FRS has been evaluated in a number of large population studies

\footnotetext{
* Correspondence: dov.shiffman@celera.com

'Celera, 1401 Harbor Bay Parkway, Alameda, CA 94502 USA

Full list of author information is available at the end of the article
}

and has been shown to predict CHD risk among individuals from different populations and a variety of ethnicities [2]. Because the FRS models were developed as sex-specific scores, the validity of FRS was typically evaluated separately in men and women [2].

Several groups have sought to improve or simplify CHD risk prediction by the FRS [3-6] by developing models that include emerging risk factors. More recently, several studies have investigated whether genetic variants associated with CHD could improve CHD risk prediction models, and much attention has been focused on a well-established genetic risk marker in the 9p21.3 locus (see Palomaki et al. [7] for a recent meta analysis). The results of these studies have been mixed. One study found that adding 9 p21.3 to a

\section{() Biomed Central}


traditional risk factor-based model improved the area under the receiver operator characteristic curve (AUC) as well as patient reclassification [8]. A second study found improvement in reclassification, but not in AUC [9]. And a third study found that $9 \mathrm{p} 21.3$ did not improve either AUC or patient reclassification [10]. Another gene variant associated with risk of $\mathrm{CHD}$ is a nonsynonymous (Trp719Arg) single nucleotide polymorphism in KIF6, which encodes a member of the kinesin superfamily. This KIF6 variant (rs20455) was associated with increased risk of CHD in the placebo groups of randomized placebo-controlled clinical trials: the secondary prevention Cholesterol and Recurrent Events (CARE) study and the primary prevention West of Scotland Coronary Prevention Study (WOSCOPS) [11]. This KIF6 variant was also associated with CHD in prospective population-based studies: Atherosclerosis Risk in Communities (ARIC) study [12], Cardiovascular Health Study [13], and the Women's Health Study [14]. Interestingly, the KIF6 variant was not associated with risk of coronary artery disease in case-control studies $[15,16]$, the explanation for the difference between the results from the prospective studies and the case control studies remains to be determined.

It has been suggested that the genetic contribution to $\mathrm{CHD}$ risk diminishes with age [17]. Since the contribution of genetic markers to CHD risk models has typically been assessed in prospective studies of middle-aged individuals, we set out to assess the contribution of genetic markers to CHD risk models in the Cardiovascular Health Study (CHS), a prospective study among Americans aged 65 or older who were followed for the occurrence of cardiovascular events [18]. Since the FRS predicts CHD risk in this population [2], CHS provided the opportunity to ask whether genetic risk markers can improve CHD risk prediction by the FRS among older North Americans. As a point of reference, we also investigated whether C-reactive protein (CRP)-a well established [19] non-genetic risk marker-can improve CHD risk prediction in the same population. Therefore, we report here the contribution of 9p21.3, KIF6 719Arg and $\mathrm{CRP}$ to CHD risk prediction in CHS.

\section{Methods}

\section{Cardiovascular Health Study}

$\mathrm{CHS}$ is a prospective observational study of risk factors for cardiovascular disease in older adults. The recruitment and design methods were previously described $[18,20]$. Briefly, men and women aged 65 years and older were recruited from random samples of Medicare eligibility lists in 4 US communities (Sacramento County, CA; Washington County, MD; Forsyth County, NC; and Pittsburgh, PA) and from age-eligible participants in the same household. Potential participants were excluded if they were institutionalized, not ambulatory at home, under hospice care, receiving radiation or chemotherapy for cancer, not expected to remain in the area for at least 3 years, or unable to be interviewed. CHS enrolled 5201 participants from 1989 to 1990; an additional 687 African American participants entered the cohort from 1992 to 1993. The CHS cohort of 5888 was $57.6 \%$ female and 15.7\% African American. The mean age at enrollment was 72.8 years (standard deviation 5.6). The institutional review board at each site approved the study methods, and all participants gave written informed consent. Collection of baseline demographic, clinical, and genetic data was previously described $[13,18,21,22]$. Briefly, participants completed a baseline clinic examination [18] that included a medical history interview, physical examination, and blood draw[21]. Baseline self-reports of MI or stroke were confirmed by information from the clinic examination or by review of medical records or physician questionnaires [22]. Genotypes of the CHS participants were determined using a multiplex method that combines polymerase chain reaction (PCR), allele-specific oligonucleotide ligation assays, and hybridization to oligonucleotides coupled to Luminex $100 \mathrm{TM} \times$ MAP microspheres (Luminex, Austin, TX) as previously described [13]. Cardiovascular events during follow-up were identified at semiannual contacts, which alternated between clinic visits and telephone calls. Suspected events were adjudicated according to standard criteria by a physician review panel using information from medical records and, in some cases, interviews with the physician, participant, or a proxy informant [23]. Medicare utilization files were searched to ascertain events that may have been missed. In this analysis, MI was defined as definite or probable nonfatal MI or definite fatal MI.

\section{Statistical analysis}

Participants with unavailable DNA or who did not consent to the use of their DNA for studies by private companies $(\mathrm{N}=514)$ were excluded from the present study. Participants for whom DNA samples were inadequate $(\mathrm{N}=130)$ were also excluded. Analyses also excluded participants who had a baseline history of MI $(\mathrm{N}=517$ of the 5244 participants), or stroke $(\mathrm{N}=222)$, who were neither white nor African American $(\mathrm{N}=30)$, or who had missing data for calculating the FRS or the three risk markers $(n=284)$. After applying these overlapping exclusions, 4284 participants remained for analysis.

We conducted analyses of time to incident MI. Follow-up began at CHS enrollment and ended on the date of incident MI, death, loss to follow-up, or June 30, 2006, whichever occurred first. The median time at risk was 12.6 years. Hazard ratios (HRs) and 95\% confidence intervals (CIs) were estimated using Cox regression models. Based on their use in the FRS, variables were 
coded as continuous (age, CRP), or categorical (smoking status, hypertension, LDL-C category, HDL-C category, diabetes, KIF6 719Arg carrier status), or ordinal (9p21.3 SNP: rs10757274).

The predicted 10-year risk of incident MI for each participant in this study was calculated based on estimates of the baseline hazard function and the regression coefficients of Cox models that included the FRS variables with or without additional risk markers as described [1]. These analyses were conducted in men and women separately as described [1,2]. The risk markers that were added to the FRS were coded continuously (CRP), additively (9p21.3 SNP), or dominantly (KIF6 719Arg) because this coding was consistent with what was reported previously for these risk markers. The change in the AUC was used to assess the improvement of the FRS by additional risk markers.

To assess whether adding risk markers to FRS resulted in reclassification of individuals to more appropriate risk categories, we used the net reclassification improvement
(NRI) measure [24]. Briefly, among those with incident events, classification is more appropriate if the individual is reclassified to a higher risk category and less appropriate if the individual is reclassified to a lower risk category. For those without incident events, the converse is true. The difference in the proportion of individuals moving to more appropriate and less appropriate categories is calculated separately for those with and without incident events. The NRI is the sum of these two differences. The predicted CHD probabilities were grouped into 10 -year risk categories of $0 \%$ to $<5 \%$, $5 \%$ to $<10 \%, 10 \%$ to $<20 \%$, and $20 \%$ or greater based on FRS models with and without additional risk markers. Statistical tests were two-sided. Data were analyzed using Stata statistical software [25].

\section{Results}

The baseline characteristics that were used to calculate the FRS among the 3651 white participants in this analysis are presented in Table 1, which also presents coefficients from

Table 1 Framingham Risk Score Characteristics at Baseline Among White Participants

\begin{tabular}{|c|c|c|c|c|}
\hline & \multicolumn{2}{|c|}{ Men $n=1495$} & \multicolumn{2}{|c|}{ Women $n=2156$} \\
\hline & Baseline Value & $\beta$ (P value)* $^{*}$ & Baseline Value & $\beta$ (P value)* $^{*}$ \\
\hline Age, y, mean (SD) & $73.3(5.7)$ & $0.043(0.0002)$ & $72.3(5.4)$ & $0.18(0.45)$ \\
\hline Current smoker & $10 \%$ & $-0.19(0.44)$ & $12 \%$ & $0.49(0.01)$ \\
\hline LDL, mg/dL, mean (SD) & $124(33)$ & NA§ & $135(37)$ & NA§ \\
\hline \multicolumn{5}{|l|}{ LDL category } \\
\hline$<100$ & $24.4 \%$ & $-0.13(0.45)$ & $16.8 \%$ & $-0.18(0.38)$ \\
\hline $100-129$ & $35.0 \%$ & reference & $30.7 \%$ & reference \\
\hline 130-159 & $28.0 \%$ & $0.10(0.49)$ & $29.4 \%$ & $-0.04(0.80)$ \\
\hline 160-189 & $10.0 \%$ & $0.22(0.29)$ & $16.2 \%$ & $0.10(0.57)$ \\
\hline$>=190$ & $2.7 \%$ & $-0.19(0.65)$ & $6.9 \%$ & $-0.23(0.41)$ \\
\hline $\mathrm{HDL}, \mathrm{mg} / \mathrm{dL}$, mean (SD) & $48(13)$ & NA§ & $59(16)$ & NA§ \\
\hline \multicolumn{5}{|l|}{ HDL category } \\
\hline$<35$ & $11.4 \%$ & $0.16(0.47)$ & $1.9 \%$ & $-0.24(0.65)$ \\
\hline $35-44$ & $34.1 \%$ & $0.090(0.60)$ & $14.2 \%$ & $0.15(0.43)$ \\
\hline $45-49$ & $18.3 \%$ & reference & $13.0 \%$ & $0.20(0.30)$ \\
\hline $50-59$ & $20.5 \%$ & $-0.21(0.29)$ & $26.9 \%$ & reference \\
\hline$>=60$ & $15.6 \%$ & $-0.030(0.89)$ & $43.9 \%$ & $-0.18(0.27)$ \\
\hline \multicolumn{5}{|l|}{ Blood pressure categoryt } \\
\hline Optimal & $23.3 \%$ & $-0.31(0.14)$ & $23.8 \%$ & $0.22(0.34)$ \\
\hline Normal & $18.5 \%$ & reference & $18.0 \%$ & reference \\
\hline high normal & $20.2 \%$ & $0.30(0.13)$ & $19.2 \%$ & $0.46(0.05)$ \\
\hline HTN stage I & $23.8 \%$ & $0.39(0.04)$ & $26.2 \%$ & $0.58(0.007)$ \\
\hline HTN stage II-IV & $14.1 \%$ & $0.21(0.33)$ & $12.8 \%$ & $0.95(0.00005)$ \\
\hline Diabetesł & $13.0 \%$ & $0.57(0.0006)$ & $8 \%$ & $0.66(0.001)$ \\
\hline
\end{tabular}

${ }^{*}$ Coefficients from Cox regression model of Framingham Risk Score (FRS) variables.

tOptimal (systolic $<120 \mathrm{~mm} \mathrm{Hg}$ and diastolic $<80 \mathrm{~mm} \mathrm{Hg}$ ), normal blood pressure (systolic 120 to $129 \mathrm{~mm} \mathrm{Hg}$ or diastolic 80 to $84 \mathrm{~mm} \mathrm{Hg}$ ), high normal blood pressure (systolic 130 to $139 \mathrm{~mm} \mathrm{Hg}$ or diastolic 85 to $89 \mathrm{~mm} \mathrm{Hg}$ ), hypertension stage I (systolic 140 to $159 \mathrm{~mm} \mathrm{Hg}$ or diastolic 90 to $99 \mathrm{~mm} \mathrm{Hg}$ ), and hypertension stage II-IV (systolic 160 or diastolic $100 \mathrm{~mm} \mathrm{Hg}$ ). When systolic and diastolic pressures fell into different categories, the higher category was selected. Blood pressure categorization was made without regard to the use of antihypertensive medication.

\#Fasting serum glucose $\geq 140$ or taking insulin or oral hypoglycemic medications.

$\S$ Continuous cholesterol measures are not included in the FRS model. 
the FRS model. We calculated the AUC for the FRS, using the coefficients estimated among men and women separately because the FRS coefficients were originally derived separately in men and women and because cardiovascular risk is generally different between men and women-men generally have $\mathrm{CHD}$ events earlier in life. The AUC among whites was 0.581 for men and 0.619 for women. After adjustment for the FRS risk factors, the risk allele $(G)$ of the 9p21.3 SNP (rs10757274) was associated with increased risk of $\mathrm{MI}$ among white men $(\mathrm{HR}=1.22 ; 95 \% \mathrm{CI} 1.03$ to $1.45, \mathrm{P}=0.02)$, this association did not reach statistical significance among white women $(\mathrm{HR}=1.16$; $95 \% \mathrm{CI} 0.98$ to $1.37, \mathrm{P}=0.08)$. Carriers of one or two 719Arg alleles of KIF6 were at increased risk of $\mathrm{MI}$ among white men $(\mathrm{HR}=$ 1.42 ; $95 \%$ CI 1.11 to $1.82, \mathrm{P}=0.006)$ but not women $(\mathrm{HR}=$ $1.05 ; 95 \% \mathrm{CI}=0.83$ to $1.33, \mathrm{P}=0.68)$. Higher $\mathrm{CRP}$ was associated with increased risk of incident $\mathrm{MI}$ among both white men $(\mathrm{HR}=1.28$ for 1 standard deviation higher CRP; $95 \%$ CI 1.14 to $1.45, \mathrm{P}=0.00005$ ) and white women $(\mathrm{HR}=1.15$; $95 \% \mathrm{CI} 1.01$ to $1.31, \mathrm{P}=0.04)$.

Among white participants, adding rs10757274 in the 9p21.3 locus to the FRS variables, did not improve the AUC among either men or women ( $P \geq 0.35$, Tables 2 and 3 ). In the net reclassification improvement (NRI) analysis, adding 9p21 to the FRS reclassified $13.7 \%$ of white men and $10.3 \%$ of white women to risk categories that were different from the FRS categories. However, the net number of individuals that were classified to more appropriate risk categories was $\leq 25$, with the NRI $\leq 0.02$ ( $P \geq 0.39$ ). Similar results were observed after CRP was added to FRS. The AUC was not improved for either white men or women $(P \geq 0.15)$ nor was the NRI significant (NRI $\leq-0.01, \mathrm{P} \geq 0.19)$. The net number of correctly reclassified individuals was $\leq 10$ in each of the two sexes. Similarly, adding KIF6 719Arg carrier status to the FRS variables did not improve risk prediction by the NRI among either men or women (NRI $\leq 0.027, \mathrm{P} \geq 0.32$, the net number of correctly reclassified individuals was $\leq 33$ ). However, adding KIF6 719Arg carrier status to the FRS modestly improved the AUC among men (from 0.581 to 0.596; $\mathrm{P}=0.03)$, though not among women $(\mathrm{P}=0.57)$.
Since the nominal value of the AUC after addition of an individual risk marker to the FRS was larger than the AUC of FRS alone (Tables 2 and 3), we asked if adding more than one marker to the FRS further improved risk prediction. Adding all three markers to the FRS resulted in the largest AUC among white men (0.612, P $=0.01$ for difference from the FRS AUC, Figure 1) and largest net number of correctly reclassified individuals $(93, \mathrm{P}=$ 0.04). However, adding all three markers to the FRS did not improve risk prediction among white women. Adding both CRP and 9p21.3 to the FRS, resulted in a marginal increase in the AUC among white men (to 0.599; $\mathrm{P}=0.09$ ), but not among white women. The NRI did not indicate improvement of risk prediction among either white men or women $(P \geq 0.4)$. In contrast, addition of KIF6 with either CRP or 9p21.3 to the FRS resulted in a larger AUC among white men (0.600 and 0.608 respectively; $\mathrm{P} \leq 0.03$ ) but not among women. The combination of KIF6 and CRP also improved risk prediction by the NRI among white men $(\mathrm{NRI}=0.093$, $\mathrm{P}=0.008$, the net number of correctly reclassified individuals $=86$ ) but not among women. However, the NRI was not significant when men and women were combined (NRI $=0.026, \mathrm{P}=0.2$ ).

For the 228 African American men and 405 women (Table 4), none of the 3 risk markers were associated with incident $\mathrm{MI}(\mathrm{P} \geq 0.05)$. The power to detect association between the genetic risk factors and MI was < $37 \%$ to detect a risk ratio of 1.5 . Thus, all results among African Americans are reported in the online supplement. Among African American participants of CHS, only 9p21.3 improved risk prediction among men as assessed by NRI (NRI $=0.182 ; \mathrm{P}=0.02$, net number of correctly reclassified individuals $=10)$. None of the other markers individually or in combination improved risk prediction by the FRS (Tables 5 and 6).

\section{Discussion}

We investigated whether adding a 9p21.3 variant, a KIF6 variant, or CRP to the FRS could improve MI risk prediction in CHS, a large prospective study of individuals

Table 2 Addition of Risk Markers to the FRS: Effect on Risk Prediction in White Men

\begin{tabular}{|c|c|c|c|c|}
\hline \multirow{2}{*}{$\begin{array}{l}\text { Markers Added } \\
\text { to FRS Model } \\
9 p 21.3\end{array}$} & \multirow{2}{*}{$\begin{array}{c}\text { Number Reclassified (\%) } \\
205(13.7)\end{array}$} & \multicolumn{2}{|c|}{$\begin{array}{l}\text { Net Number Reclassified } \\
\text { Correctly (NRI, P value) }\end{array}$} & \multirow{2}{*}{$\begin{array}{c}\text { AUC (P value)* } \\
0.586(0.35)\end{array}$} \\
\hline & & 25 & $(0.021,0.41)$ & \\
\hline KIF6 & 309 (20.7) & 33 & $(0.027,0.32)$ & $0.596(0.03)$ \\
\hline CRP & $346(23.1)$ & 10 & $(-0.017,0.61)$ & $0.595(0.15)$ \\
\hline CRP $+9 p 21.3$ & $412(27.6)$ & 56 & $(0.029,0.40)$ & 0.599 (0.09) \\
\hline CRP + KIF6 & 466 (31.2) & 86 & $(0.093,0.008)$ & $0.608(0.02)$ \\
\hline $9 p 21.3+$ KIF6 & $348(23.3)$ & 40 & $(0.041,0.18)$ & $0.600(0.03)$ \\
\hline CRP + 9p21.3 + KIF6 & $495(33.1)$ & 93 & $(0.076,0.04)$ & $0.612(0.01)$ \\
\hline
\end{tabular}

*P value for change in the AUC from the AUC of the FRS model (0.581). 
Table 3 Addition of Risk Markers to the FRS: Effect on Risk Prediction in White Women

\begin{tabular}{lcccc}
\hline Markers Added to FRS Model & Number Reclassified (\%) & \multicolumn{2}{c}{$\begin{array}{c}\text { Net Number Reclassified } \\
\text { Correctly (NRI, P value) }\end{array}$} & $\begin{array}{c}\text { AUC (P value)* } \\
\text { 9p21.3 }\end{array}$ \\
KIF6 & $222(10.3)$ & -16 & $(-0.018,0.39)$ & $0.621(0.57)$ \\
CRP & $72(3.3)$ & 0 & $(0.007,0.61)$ & $0.618(0.57)$ \\
CRP + 9p21.3 & $270(12.5)$ & -10 & $(-0.032,0.19)$ & $0.617(0.69)$ \\
CRP + KIF6 & $362(16.8)$ & 20 & $(0.007,0.81)$ & $0.620(0.81)$ \\
$9 p 21.3+$ KIF6 & $276(12.8)$ & -4 & $(-0.029,0.24)$ & $0.617(0.67)$ \\
CRP + 9p21.3 + KIF6 & $246(11.4)$ & -2 & $(0.015,0.52)$ & $0.620(0.65)$ \\
\hline
\end{tabular}

*P value for change in the AUC from the AUC of the FRS model (0.619).

aged 65 years or older from American communities. Adding individual risk markers had, at best, a modest effect on risk prediction. Adding two or more risk markers, specifically risk marker combinations that included the KIF6 719Arg genotype, resulted in a somewhat higher, though still modest improvement in risk prediction as measured among white males by either the AUC or by NRI, but not in the combined male and female population.

The appropriate methods for assessing improvement of risk prediction by risk markers have been widely debated [26]. And given the limitations of the AUC measure, alternative assessment methods such as NRI have been proposed [24]. Although the current study was not designed to compare the AUC and NRI, the results from these two measures were largely consistent.

The ability of CRP to improve risk stratification has been evaluated in multiple studies, which found that the addition of CRP to risk prediction models provided modest improvement in risk prediction (see Buckley et al. [19] and Schnell-Inderst et al. [27] for recent

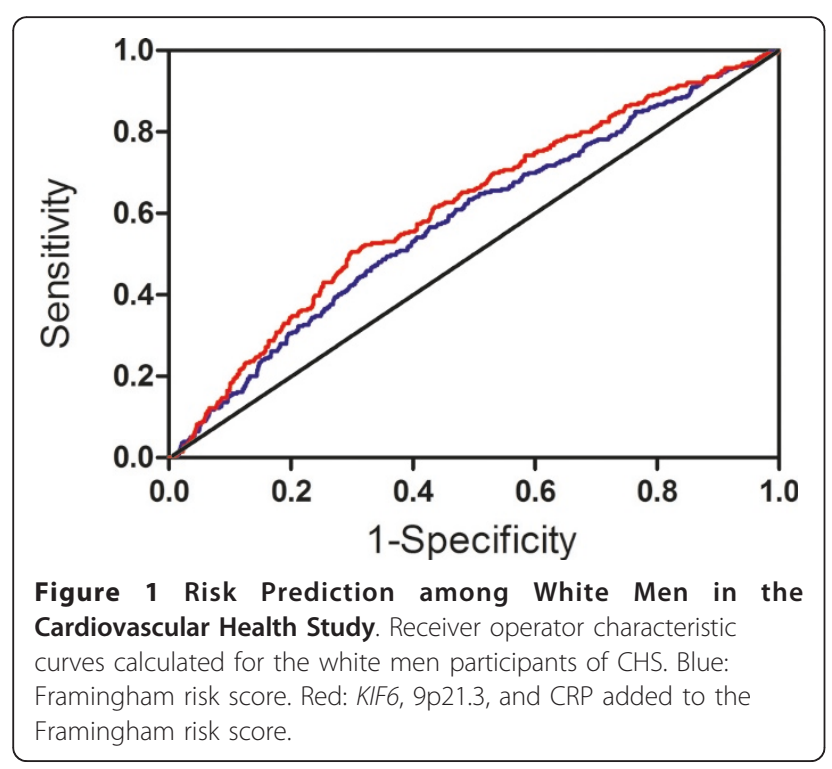

comprehensive evaluations). In CHS, CRP did not improve risk prediction by the FRS as assessed by the AUC or NRI measures, consistent with previously published observations that the association of a risk factor with MI after adjustment for the FRS variables does not necessarily mean that the risk factor will improve risk prediction beyond the FRS, as measured by the change in the AUC, partly because even risk factors with large odds ratio (up to 7) have distributions that overlap substantially between those with and without disease $[28,29]$. However, the addition of CRP in combination with KIF6 719Arg to the FRS improved risk prediction by both the AUC and NRI measures among white men.

Although the association between CHD and SNPs in the 9p21.3 locus has been reported extensively [7], Dehghan et al. [30] reported that SNPs in this locus were not associated with risk of CHD in a prospective study of older Europeans. In contrast, we have observed in CHS, a population of individuals 65 years and older, that a SNP in the 9p21.3 locus is associated with MI among men, although this association did not reach statistical significance among women. We have also observed in CHS an association between MI and KIF6 719Arg carrier status among men but not among women. However, published results from the Women's Health Study [10,14] suggest that both 9p21.3 and KIF6 719Arg are associated with MI among women. These inconsistent findings among women could be due to lack of power in CHS, or to different baseline risk of MI in CHS and the Women's Health Study. Additional analysis of the 9p21.3 and KIF6 variants in prospective studies of women would be required to understand this apparent inconsistency.

The addition of the 9p21.3 variant to the FRS did not improve risk prediction in CHS as measured by either the AUC or NRI among whites. Similarly, addition of a SNP in the 9 p21.3 locus did not improve risk prediction by traditional risk factors in the Women's Health Study [10]. However, in ARIC [8] the addition of 9p21.3 to the FRS resulted in a modest but statistically significant improvement in risk prediction as measured by AUC or 
Table 4 Framingham Risk Score Characteristics at Baseline among Black Participants

\begin{tabular}{|c|c|c|c|c|}
\hline & \multicolumn{2}{|c|}{ Men $n=228$} & \multicolumn{2}{|c|}{ Women $n=405$} \\
\hline & Baseline Value & $\beta$ (P value)* $^{*}$ & Baseline Value & $\beta$ (P value)* \\
\hline Age, $y$, mean (SD) & $72.3(5.5)$ & $0.035(0.33)$ & $73.0(5.6)$ & $1.37(0.05)$ \\
\hline Current smoker & $23 \%$ & $0.28(0.55)$ & $14 \%$ & $0.0085(0.99)$ \\
\hline LDL, mg/dL, mean (SD) & $124(32)$ & NA§ & $132(37)$ & NA§ \\
\hline \multicolumn{5}{|l|}{ LDL category } \\
\hline$<100$ & $22.4 \%$ & $0.36(0.46)$ & $17.8 \%$ & $1.08(0.02)$ \\
\hline $100-129$ & $34.2 \%$ & reference & $33.3 \%$ & reference \\
\hline $130-159$ & $31.6 \%$ & $-0.34(0.51)$ & $29.4 \%$ & $0.67(0.13)$ \\
\hline $160-189$ & $9.6 \%$ & $0.92(0.11)$ & $12.6 \%$ & $0.52(0.33)$ \\
\hline$>=190$ & $2.2 \%$ & $0.066(0.95)$ & $6.9 \%$ & $1.20(0.02)$ \\
\hline $\mathrm{HDL}, \mathrm{mg} / \mathrm{dL}$, mean (SD) & $53(14)$ & & $61(15)$ & \\
\hline \multicolumn{5}{|l|}{ HDL category } \\
\hline$<35$ & $4.4 \%$ & $-0.22(0.84)$ & $0.7 \%$ & \\
\hline $35-44$ & $23.2 \%$ & $-0.75(0.15)$ & 11.85 & $-0.43(0.45)$ \\
\hline $45-49$ & $21.5 \%$ & reference & $9.9 \%$ & $-0.18(0.76)$ \\
\hline $50-59$ & $24.1 \%$ & $-1.06(0.05)$ & $29.9 \%$ & reference \\
\hline$>=60$ & $26.8 \%$ & $-0.69(0.16)$ & $47.6 \%$ & $-0.17(0.64)$ \\
\hline \multicolumn{5}{|l|}{ Blood pressure category $\dagger$} \\
\hline Optimal & $17.1 \%$ & $-0.46(0.52)$ & $13.6 \%$ & $0.59(0.50)$ \\
\hline Normal & $17.5 \%$ & reference & $14.1 \%$ & reference \\
\hline high normal & $19.3 \%$ & $0.080(0.89)$ & $18.0 \%$ & $0.81(0.32)$ \\
\hline HTN stage I & $32.0 \%$ & $0.34(0.52)$ & $31.1 \%$ & $1.01(0.18)$ \\
\hline HTN stage II-IV & $14.0 \%$ & $0.67(0.29)$ & $23.2 \%$ & $1.55(0.04)$ \\
\hline Diabetesł & $19 \%$ & $-0.15(0.76)$ & $20 \%$ & $0.51(0.17)$ \\
\hline
\end{tabular}

*Coefficients from Cox regression model of Framingham Risk Score (FRS) variables.

tOptimal (systolic < $120 \mathrm{~mm} \mathrm{Hg}$ and diastolic < $80 \mathrm{~mm} \mathrm{Hg}$ ), normal blood pressure (systolic 120 to $129 \mathrm{~mm} \mathrm{Hg}$ or diastolic 80 to $84 \mathrm{~mm} \mathrm{Hg}$ ), high normal blood pressure (systolic 130 to $139 \mathrm{~mm} \mathrm{Hg}$ or diastolic 85 to $89 \mathrm{~mm} \mathrm{Hg}$ ), hypertension stage I (systolic 140 to $159 \mathrm{~mm} \mathrm{Hg}$ or diastolic 90 to $99 \mathrm{~mm} \mathrm{Hg}$ ), and hypertension stage II-IV (systolic 160 or diastolic $100 \mathrm{~mm} \mathrm{Hg}$ ). When systolic and diastolic pressures fell into different categories, the higher category was selected. Blood pressure categorization was made without regard to the use of antihypertensive medication.

†Fasting serum glucose $\geq 140$ or taking insulin or oral hypoglycemic medications.

$\S$ Continuous cholesterol measures are not included in the FRS model.

reclassification, and in the Northwick Park Heart Study II [9] adding 9p21.3 improved reclassification but not AUC. These inconsistent results could be attributed to differences in baseline risk between the studies, (the older CHS population is likely to have greater baseline risk of MI), which could affect the power to detect the modest improvement in risk prediction contributed by the $9 \mathrm{p} 21.3$ variant.
Genetic and non-genetic biomarkers offer different benefits in the assessment of CHD risk. Non-genetic biomarkers could change over time, and therefore, repeat measurements may be necessary because of dayto-day variation in the level of these biomarkers. However, repeat measurements of non-genetic biomarkers may also provide an indication of successful medical

Table 5 Addition of Risk Markers to the FRS: Effect on Risk Prediction in Black Men

\begin{tabular}{|c|c|c|c|}
\hline $\begin{array}{l}\text { Markers Added } \\
\text { to FRS Model }\end{array}$ & Number Reclassified (\%) & $\begin{array}{l}\text { Net Number Reclassified } \\
\text { Correctly (NRI, P value) }\end{array}$ & AUC (P value)* \\
\hline $9 p 21$ & $50(21.9)$ & $10(0.182,0.02)$ & $0.688(0.07)$ \\
\hline KIF6 & no data† & no datat & no data† \\
\hline CRP & $48(21.1)$ & $-4(0.006,0.94)$ & $0.654(0.92)$ \\
\hline$C R P+9 p 21$ & $70(30.7)$ & $10(0.129,0.16)$ & $0.675(0.51)$ \\
\hline CRP + KIF6 & no data† & no data† & no data† \\
\hline $9 p 21+$ KIF6 & no data† & no data† & no data† \\
\hline CRP $+9 p 21+$ KIF6 & no data† & no data† & no data† \\
\hline
\end{tabular}

*P value for change in AUC from AUC of the FRS model (0.657).

tRisk for KIF6 cannot be calculated because none of the 15 African American men who were noncarriers of the KIF6 719Arg risk allele had an event. 
Table 6 Addition of Risk Markers to the FRS: Effect on Risk Prediction in Black Women

\begin{tabular}{lccc}
\hline $\begin{array}{l}\text { Markers Added } \\
\text { to FRS Model }\end{array}$ & Number Reclassified (\%) & $\begin{array}{l}\text { Net Number Reclassified } \\
\text { Correctly (NRI, P value) }\end{array}$ & AUC (P value)* \\
\hline 9p21 & $110(27.2)$ & $12(0.013,0.89)$ & $0.734(0.40)$ \\
KIF6 & $11(2.7)$ & $-1(-0.023,0.35)$ & $0.723(0.94)$ \\
CRP & $31(7.7)$ & $13(0.016,0.70)$ & $0.726(0.63)$ \\
CRP + 9p21 & $118(29.1)$ & $14(0.039,0.70)$ & $0.735(0.39)$ \\
CRP + KIF6 & $33(8.1)$ & $9(0.025,0.60)$ & $0.725(0.83)$ \\
9p21 + KIF6 & $113(27.9)$ & $15(0.042,0.66)$ & $0.733(0.46)$ \\
CRP + 9p21 + KIF6 & $119(29.3)$ & $15(0.002,0.99)$ & $0.734(0.46)$ \\
\hline
\end{tabular}

*P value for change in AUC from AUC of the FRS model (0.723).

therapy or life-style modification. Genetic biomarkers do not change and thus need only be measured once to obtain information about the lifelong exposure to that biomarker. The 9p21.3 and KIF6 gene variants were chosen for investigation because they have both been reported to be associated with CHD in multiple prospective studies and are common variants. For example, in the white population about $75 \%$ of carry at least one risk allele of 9 p 21.3 and about $65 \%$ carry at least one KIF6 719Arg risk allele. CRP was chosen because of the well-established association between CRP levels and risk of CHD and because of continuing interest in whether it should be added to risk prediction algorithms. Elevated CRP is also common. For example, although CRP was analyzed as a continuous variable in this study in order to increase the power of the study, others have reported that in CHS, $26 \%$ of the population have elevated CRP (> $3 \mathrm{mg} / \mathrm{dL}$ ) [31]. Thus, $\sim 17 \%$ of the CHS white population have both elevated CRP and carry the 719Arg allele of KIF6.

This study has several limitations. The AUC of the FRS model for white men (0.581) and white women (0.619) in this study of older individuals is lower than the AUC that has been reported for middle age populations (e.g., 0.75 and 0.83 among white men and among white women in ARIC [2]), thus the markers we studied may only improve risk prediction in populations in which the ability of the FRS to predict CHD is modest. The difference observed between the AUC and NRI measures for the addition of KIF6 719Arg among men could be attributed to the overall modest improvement of risk prediction by single marker addition. Another limitation of this study is the limited number of genetic markers evaluated-a recent paper suggested that risk prediction models might be improved by incorporating large number of genetic markers into a genetic risk score [32]. Lastly, the effect of these single and multiple marker additions on risk prediction was investigated in a population of individuals aged 65 or older at baseline and our observations may not be generalizable to younger populations.

\section{Conclusions}

In the white male population of $\mathrm{CHS}$, the addition of KIF6 719Arg in combination with 9p21.3, CRP, or both modestly improved risk prediction. This improvement was not significant after multiple-testing correction and was not observed in the combined male and female population.

\section{Sources of Funding}

The research reported in this article was supported by contracts N01-HC-15103, N01-HC-35129, N01-HC-45133, N01-HC-55222, N01-HC-75150, N01-HC-85079 through N01-HC-85086, and U01 HL080295 from the National Heart, Lung, and Blood Institute, with additional contribution from the National Institute of Neurological Disorders and Stroke. R.P.T. was supported by NIH RO1 HL077499.

\section{Acknowledgements}

The authors thank the CHS participants. A full list of participating CHS investigators and institutions can be found at http://www.chs-nhlbi.org.

\section{Author details}

${ }^{1}$ Celera, 1401 Harbor Bay Parkway, Alameda, CA 94502 USA. ${ }^{2}$ Group Health Research Institute, Seattle, WA 98101 USA. ${ }^{3}$ Department of Medicine and Pathology, University of Vermont, Colchester Research Facility, Colchester VT 05446 USA. ${ }^{4}$ Departments of Pathology and Biochemistry, College of Medicine, University of Vermont, Burlington, VT 05405-0068 USA.

${ }^{5}$ Departments of Medicine, Epidemiology, and Health Services, University of Washington, Seattle, WA 98115 USA.

\section{Authors' contributions}

Study design: DS, MC, RPT, JJD, BMP. Data collection: JZL, MC, BMP. Data analysis: ESO, JZL, CMR. Manuscript writing: DS, ESO, CMR, MC, RPT, JJD, BMP. All authors read and approved the final manuscript.

\section{Conflict of Interest}

DS, CMR, JZL, and JJD are employees of Celera.

Received: 26 January 2011 Accepted: 15 March 2011

Published: 15 March 2011

\section{References}

1. Wilson PWF, D'Agostino RB, Levy D, Belanger AM, Silbershatz H, Kannel WB: Prediction of coronary heart disease using risk factor categories. Circulation 1998, 97:1837-1847.

2. D'Agostino RB, Grundy S, Sullivan LM, Wilson P: Validation of the Framingham coronary heart disease prediction scores: results of a multiple ethnic groups investigation. JAMA 2001, 286:180-7. 
3. De Backer G, Ambrosioni E, Borch-Johnsen K, Brotons C, Cifkova R, Dallongeville J, Ebrahim S, Faergeman O, Graham I, Mancia G, et al: European guidelines on cardiovascular disease prevention in clinical practice. European Heart Journal 2004, 173:379-389.

4. Folsom AR, Chambless LE, Ballantyne CM, Coresh J, Heiss G, Wu KK, Boerwinkle E, Mosley TH, Sorlie P, Diao G, et al: An assessment of incremental coronary risk prediction using C-reactive protein and other novel risk markers: the atherosclerosis risk in communities study. Archives of internal medicine 2006, 166:1368-1373.

5. Ridker PM, Buring JE, Rifai N, Cook NR: Development and validation of improved algorithms for the assessment of global cardiovascular risk in women: the Reynolds Risk Score. JAMA 2007, 297:611-619.

6. Assmann G, Cullen P, Schulte H: Simple scoring scheme for calculating the risk of acute coronary events based on the 10-year follow-up of the prospective cardiovascular Münster (PROCAM) study. Circulation 2002, 105:310-315.

7. Palomaki GE, Melillo S, Bradley LA: Association between 9p21 genomic markers and heart disease: a meta-analysis. JAMA 2010, 303:648-656.

8. Brautbar A, Ballantyne CM, Lawson K, Nambi V, Chambless L, Folsom AR, Willerson JT, Boerwinkle E: Impact of adding a single allele in the 9p21 locus to traditional risk factors on reclassification of coronary heart disease risk and implications for lipid-modifying therapy in the Atherosclerosis Risk in Communities study. Circulation. Cardiovascular genetics 2009, 2:279-285.

9. Talmud PJ, Cooper JA, Palmen J, Lovering R, Drenos F, Hingorani AD, Humphries SE: Chromosome 9p21.3 coronary heart disease locus genotype and prospective risk of CHD in healthy middle-aged men. Clinical chemistry 2008, 54:467-474.

10. Paynter NP, Chasman DI, Buring JE, Shiffman D, Cook NR, Ridker PM: Cardiovascular disease risk prediction with and without knowledge of genetic variation at chromosome 9p21. 3. Annals of internal medicine 2009, 150:65-72.

11. lakoubova OA, Tong $\mathrm{CH}$, Rowland CM, Kirchgessner TG, Young BA, Arellano AR, Shiffman D, Sabatine MS, Campos H, Packard CJ, et al: Association of the Trp719Arg polymorphism in kinesin-like protein 6 with myocardial infarction and coronary heart disease in 2 prospective trials: the CARE and WOSCOPS trials. Journal of the American College of Cardiology 2008, 51:435-443.

12. Bare LA, Morrison AC, Rowland CM, Shiffman D, Luke MM, lakoubova OA, Kane JP, Malloy MJ, Ellis SG, Pankow JS, et al: Five common gene variants identify elevated genetic risk for coronary heart disease. Genetics in medicine 2007, 9:682-689.

13. Shiffman D, O'Meara ES, Bare LA, Rowland CM, Louie JZ, Arellano AR, Lumley T, Rice K, lakoubova OA, Luke MM, et al: Association of gene variants with incident myocardial infarction in the Cardiovascular Health Study. Arteriosclerosis, thrombosis, and vascular biology 2008, 28:173-179.

14. Shiffman D, Chasman DI, Zee RYL, lakoubova OA, Louie JZ, Devlin JJ, Ridker PM: A kinesin family member 6 variant is associated with coronary heart disease in the Women's Health Study. Journal of the American College of Cardiology 2008, 51:444-448.

15. Stewart AFR, Dandona S, Chen L, Assogba O, Belanger M, Ewart G, LaRose R, Doelle H, Williams K, Wells GA, et al: Kinesin family member 6 variant Trp719Arg does not associate with angiographically defined coronary artery disease in the Ottawa Heart Genomics Study. Journal of the American College of Cardiology 2009, 53:1471-1472.

16. Assimes TL, Hólm H, Kathiresan S, Reilly MP, Thorleifsson G, Voight BF, Erdmann J, Willenborg C, Vaidya D, Xie C, et al: Lack of association between the Trp719Arg polymorphism in kinesin-like protein- 6 and coronary artery disease in 19 case-control studies. Journal of the American College of Cardiology 2010, 56:1552-1563.

17. Zdravkovic S, Wienke A, Pedersen NL, Marenberg ME, Yashin Al, De Faire U: Heritability of death from coronary heart disease: a 36-year follow-up of 20966 Swedish twins. Journal of internal medicine 2002, 252:247-254.

18. Fried LP, Borhani NO, Enright P, Furberg CD, Gardin JM, Kronmal RA, Kuller LH, Manolio TA, Mittelmark MB, Newman A: The Cardiovascular Health Study: design and rationale. Ann Epidemiol 1991, 1:263-276.

19. Buckley DI, Fu R, Freeman M, Rogers K, Helfand M: C-reactive protein as a risk factor for coronary heart disease: a systematic review and metaanalyses for the US Preventive Services Task Force. Annals of internal medicine 2009, 151:483-495.
20. Tell GS, Fried LP, Hermanson B, Manolio TA, Newman AB, Borhani NO: Recruitment of adults 65 years and older as participants in the Cardiovascular Health Study. Annals of epidemiology 1993, 3:358-366.

21. Cushman M, Cornell ES, Howard PR, Bovill EG, Tracy RP: Laboratory methods and quality assurance in the Cardiovascular Health Study. Clinical chemistry 1995, 41:264-70.

22. Ives DG, Fitzpatrick AL, Bild DE, Psaty BM, Kuller LH, Crowley PM, Cruise RG, Theroux S: Surveillance and ascertainment of cardiovascular events: The Cardiovascular Health Study. Annals of Epidemiology 1995, 5:278-285.

23. Psaty BM, Kuller LH, Bild D, Burke GL, Kittner SJ, Mittelmark M, Price TR, Rautaharju PM, Robbins J: Methods of assessing prevalent cardiovascular disease in the Cardiovascular Health Study. Annals of Epidemiology 1995, 5:270-277.

24. Pencina MJ, D'Agostino RB Sr, D'Agostino RB Jr, Vasan RS: Evaluating the added predictive ability of a new marker: from area under the ROC curve to reclassification and beyond. Statistics in medicine 2008, 27:157-172.

25. StataCorp: Stata Statistical Software: Release 9. 2005.

26. Hlatky Ma, Greenland P, Arnett DK, Ballantyne CM, Criqui MH, Elkind MSV, Go AS, Harrell FE, Hong Y, Howard BV, et al: Criteria for evaluation of novel markers of cardiovascular risk: a scientific statement from the American Heart Association. Circulation 2009, 119:2408-2416.

27. Schnell-Inderst P, Schwarzer R, Göhler A, Grandi N, Grabein K, Stollenwerk B, Manne J, Klauss V, Siebert U, Wasem J: Prognostic value, clinical effectiveness, and cost-effectiveness of high-sensitivity C-reactive protein as a marker for major cardiac events in asymptomatic individuals: a health technology assessment report. International journal of technology assessment in health care 2010, 26:30-39.

28. Pepe MS, Janes $H$, Longton $G$, Leisenring W, Newcomb P: Limitations of the odds ratio in gauging the performance of a diagnostic, prognostic, or screening marker. American Journal of Epidemiology 2004, 159:882-890.

29. Psaty BM: Drug therapies for the primary prevention of cardiovascular events: trials and errors: 2009 Ancel Keys Memorial Lecture. Circulation 2010, 121:940-945

30. Dehghan A, van Hoek M, Sijbrands EJG, Oostra BA, Hofman A, van Duijn CM, Witteman JCM: Lack of association of two common polymorphisms on 9p21 with risk of coronary heart disease and myocardial infarction; results from a prospective cohort study. BMC medicine 2008, 6:30.

31. Cushman M, Arnold AM, Psaty BM, Manolio TA, Kuller LH, Burke GL, Polak JF, Tracy RP: C-reactive protein and the 10-year incidence of coronary heart disease in older men and women: the cardiovascular health study. Circulation 2005, 112:25-31.

32. Davies RW, Dandona S, Stewart AFR, Chen L, Ellis SG, Tang WHW, Hazen SL, Roberts R, McPherson R, Wells GA: Improved prediction of cardiovascular disease based on a panel of single nucleotide polymorphisms identified through genome-wide association studies. Circulation. Cardiovascular genetics 2010, 3:468-474.

\section{Pre-publication history}

The pre-publication history for this paper can be accessed here: http://www.biomedcentral.com/1471-2261/11/10/prepub

doi:10.1186/1471-2261-11-10

Cite this article as: Shiffman et al:: The contribution of a 9p21.3 variant, a KIF6 variant, and C-reactive protein to predicting risk of myocardial infarction in a prospective study. BMC Cardiovascular Disorders 2011 11:10. 